\title{
Characterization of thin polymer films using terahertz time-domain interferometry
}

\author{
S. Krishnamurthy, M. T. Reiten, S. A. Harmon, and R. A. Cheville ${ }^{\text {a) }}$ \\ School of Electrical and Computer Engineering and Center for Laser and Photonic Research, \\ Oklahoma State University, Stillwater, Oklahoma 74078
}

(Received 28 March 2001; accepted for publication 4 June 2001)

\begin{abstract}
An interferometer for broadband single-cycle $\mathrm{THz}$ pulses is developed based on the Michelson configuration. Total internal reflection of $\mathrm{THz}$ pulses in high-resistivity silicon prisms provides a nearly $180^{\circ}$ phase shift of one arm relative to the other to achieve destructive interference. We show that due to automatic compensation for laser fluctuations by the interferometer, it is possible to measure the index and absorption of thin-film samples with more accuracy than is achievable with standard THz time-domain spectroscopy. We demonstrate characterization of the complex index of refraction of $2 \mu \mathrm{m}$ thick Mylar (polyester) films. By measuring the signal amplitude directly in the time domain, the interferometer can be used for rapid measurements of film thickness with a resolution of better than $1 \mu \mathrm{m}$. C 2001 American Institute of Physics. [DOI: 10.1063/1.1389823]
\end{abstract}

The measurement of thin films ${ }^{1}$ is one of the enabling roles of optics in modern day industry. ${ }^{2}$ Accurate measurement and control of film thickness are critical to applications from semiconductor processing to food packaging. However, well developed optical techniques such as variable angle spectroscopic ellipsometry and phase-contrast microscopy are not applicable for measurement of opaque films.

The recently developed techniques of $\mathrm{THz}$ time-domain spectroscopy (THz TDS) ${ }^{1}$ and THz tomography $y^{3,4}$ have been shown to be capable of performing measurements on, and images of, samples which are opaque in the visible and nearinfrared regions of the spectrum. The utility of THz TDS is limited in the case in which the sample is very thin, or has a low index and absorption, since it is very difficult to distinguish changes in the $\mathrm{THz}$ pulse caused by the sample from those caused by long term fluctuations in the driving laser source or experiment. Using standard THz TDS, $172 \mu \mathrm{m}$ films of polyimide have been characterized. ${ }^{5}$ In transmission measurements, rapid sample dithering has permitted differential measurements of parylene- $\mathrm{N}$ films. ${ }^{6}$ An interferometer incorporating the Gouy phase shift has permitted reflection measurements of voids in Teflon as small as $12.5 \mu \mathrm{m} .^{7}$

The experimental setup consists of a Michelson type interferometer configuration inserted into a standard THz TDS system as shown in Fig. 1. The $\mathrm{THz}$ source is a coplanar transmission line biased at $40 \mathrm{~V}_{\mathrm{dc}}$ which generates freely propagating pulses of $\mathrm{THz}$ electromagnetic radiation. ${ }^{8}$ The radiation emitted covers a $0.3-1.5 \mathrm{THz}$ bandwidth [see the reference spectrum in Fig. 3(b)] and is collected by a silicon lens and collimated by a parabolic mirror. The $\mathrm{THz}$ beam (shown by thick lines) is split by a $0.35 \mathrm{~mm}$ thick silicon beam splitter into reference and sample pulse trains. The two $\mathrm{THz}$ pulse trains are incident on silicon right angle prisms which act as roof reflectors. One face of the sample arm prism is coated with a $60 \mathrm{~nm}$ thick layer of aluminum deposited by thermal evaporation. After passing through the prisms, the separate $\mathrm{THz}$ pulses are again made coincident

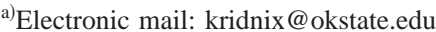

by the second beam splitter. A second parabolic mirror collimates the superposition of the two $\mathrm{THz}$ pulse trains which are then incident on the $50 \mu \mathrm{m}$ receiver antenna. Thin-film samples are placed in the sample arm in the position shown in Fig. 1.

In THz TDS, a sample with complex refractive index, $n(\omega)=n_{r}(\omega)-i n_{i}(\omega)$, is placed in the THz beam and the electric field $E_{\text {samp }}(t)$ transmitted through, ${ }^{1}$ or reflected from, ${ }^{9}$ the sample is measured. The sample is then removed, and a second reference scan is measured, $E_{\text {ref }}(t)$. The numerical Fourier transform of the time dependent data yields the complex amplitude spectra $E_{\text {samp }}(\omega)$ and $E_{\text {ref }}(\omega)$. The ratio of the sample spectrum to the reference spectrum determines the phase and amplitude shift of the THz pulse interacting with the sample. Assuming a transmitted pulse going through a sample of thickness $d$

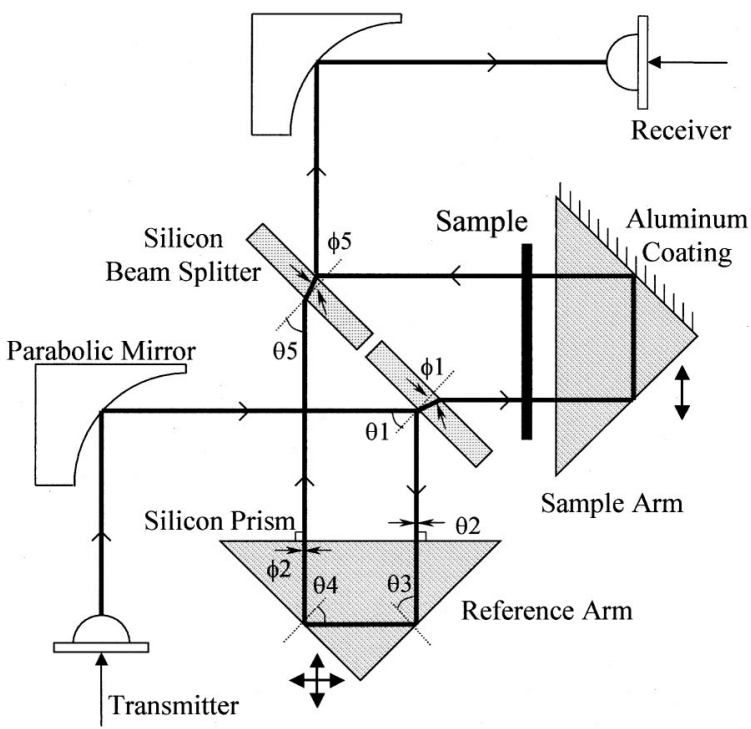

FIG. 1. Diagram of the THz interferometer. The beam splitters are independently adjustable and the prisms can be translated into the directions indicated by the arrows. The incidence angle on the prism faces $\left(\theta_{2}\right.$ and $\left.\phi_{2}\right)$ is $0^{\circ}$. 


$$
\frac{E_{\text {samp }}(\omega)}{E_{\text {ref }}(\omega)}=\exp \left\{-i k_{0}\left[n_{r}(\omega)-1-i n_{i}(\omega)\right] d\right\},
$$

with $k_{0}=\omega / c$. With the power absorption coefficient given by $\alpha=2 \omega n_{i}(\omega) / c$, both the real part of the refractive index and the absorption spectra are determined from the ratio of the measurements. For samples with high index the Fresnel transmission coefficients, $t_{12}(\omega)$ and $t_{21}(\omega)$, on the input and output faces, respectively, must be included. ${ }^{10}$

In the THz TDS system shown in Fig. 1, the THz pulse is split into both sample, $E_{\text {samp }}(t)$, and reference, $E_{\text {ref }}(t)$, pulses and a single measurement returns the superposition $E(t)=E_{\text {samp }}(t)+E_{\text {ref }}(t)$. The measured fields from each arm are then given by the incident field multiplied by the Fresnel coefficients along the beam path. The Fresnel coefficients ${ }^{11}$ are defined as $r_{12}^{\theta}$ for reflection and $t_{12}^{\theta}$ for transmission where the subscript " 12 " corresponds to an air-silicon interface and "21" to a silicon-air interface at incident angle $\theta$. The Fresnel coefficient $r_{2 M}^{\theta 4}$ is the reflection coefficient at the silicon-metal interface. By rotating the prisms to minimize the time delay, the interferometer is aligned so $\theta_{3}=45^{\circ}$ and $\theta_{2}=\theta_{4}=0^{\circ}$ and for this optimal alignment $\theta_{1}=\theta_{5}=45^{\circ}$. Contrary to intuition, the silicon-metal reflection, $r_{2 M}^{\theta 4}$, has a value of +1 when the field is polarized in the plane of incidence $(P) .{ }^{11}$ The complex coefficient $r_{21}^{45^{\circ}}=-0.983+0.186 i$ corresponds to total internal reflection with $\left|r_{21}^{45^{\circ}}\right|=1$ with a phase angle of $169.7^{\circ}$. The sample transmission function, $T_{s}(\omega)$ is given by

$$
T_{s}(\omega)=t_{1 S} t_{S 1} \exp \left\{-i k_{0}\left[n_{r}(\omega)-1-i n_{i}(\omega)\right] d\right\},
$$

where $t_{1 S}$ and $t_{S 1}$ are the Fresnel coefficients for $\theta=0^{\circ}$ and we assume the sample index is small so secondary reflections can be ignored.

With the reference arm phase defined to be $0^{\circ}$ and $T_{s}(\omega)=1, \quad E_{\mathrm{ref}}(\omega)=0.24 E_{0}(\omega) \quad$ and $\quad E_{\text {samp }}(\omega)$ $=-0.24 E_{0}(\omega) T_{s}(\omega) \exp \left(i 10.3^{\circ}\right)$. This phase shift arises from the frequency independent Fresnel reflection coefficient which has complex value for total internal reflection. The temporal phase shift has been observed previously with $\mathrm{THz}$ pulses. ${ }^{12}$ Due to this phase shift between the arms, even under optimal alignment conditions complete destructive interference of the $\mathrm{THz}$ wave forms is impossible to achieve. A numerical analysis for this system shows that the best extinction possible in the time domain is approximately $2.5 \%$ of the signal propagated by one arm.

The measured pulses in the reference arm, $E_{\text {ref }}(t)$, and sample arm, $E_{\text {samp }}(t)$, obtained by blocking one arm and measuring the signal in the other arm are shown in Fig. 2(a). The measured signal (points) in the sample arm is also shown when a $2 \mu \mathrm{m}$ thick Mylar ${ }^{\circledR}$ sample is placed in this arm. The small value of $k_{0}(n-1) d$ makes these two $\mathrm{THz}$ pulses nearly indistinguishable. Figure 2(b) shows the measured interferometer signal with both the sample and reference arms open. The measured peak to peak signal here is $3.5 \%$ of the difference between the peaks of the reference and sample arms measured individually. The slight additional signal over the $2.5 \%$ calculated minimum is attributed to slight misalignments of the $\mathrm{THz}$ beam. The signal measured with both arms open and a nominally $2 \mu \mathrm{m}$ Mylar film as a sample, $E_{\text {meas }}(t)$, is shown in Fig. 2(b).

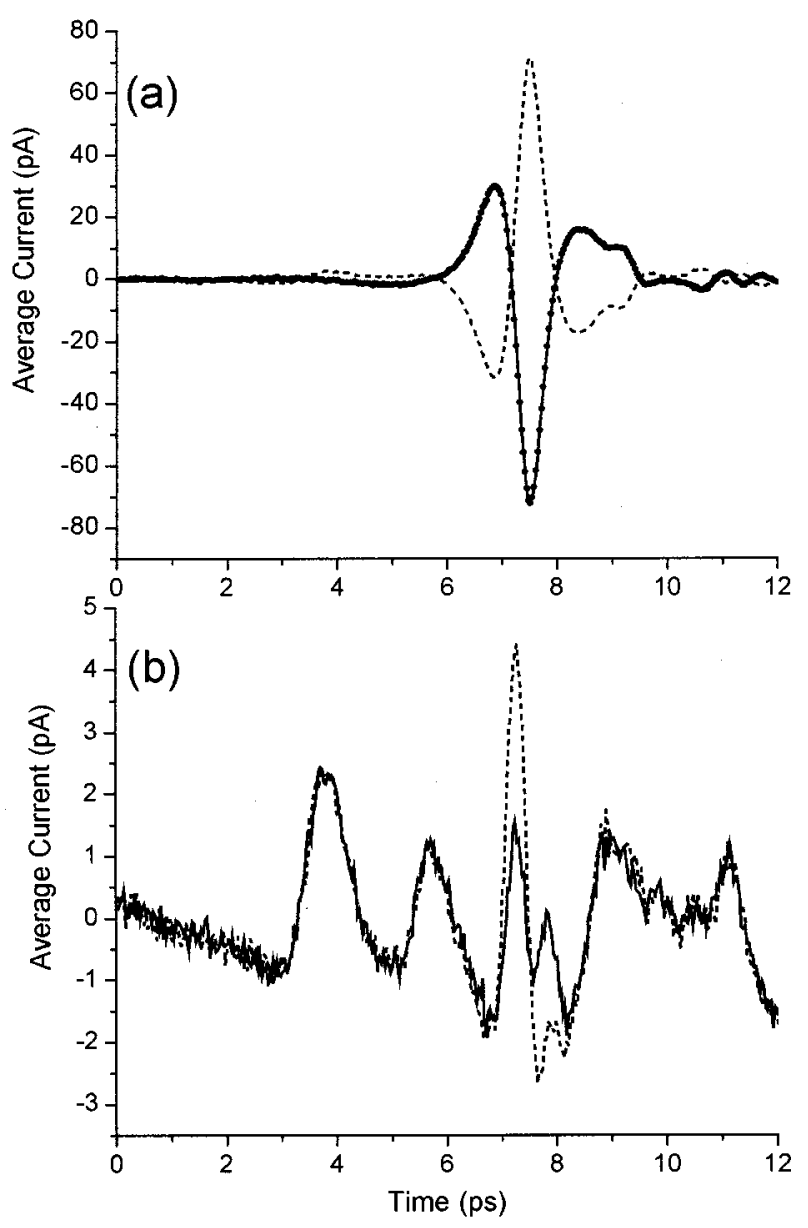

FIG. 2. Measurement of the $\mathrm{THz}$ signal in the (a) reference arm (dashed line) and sample arm (solid line). The dotted line represents the sample arm when the sample is placed in the arm. (b) The measured signal with no sample in the beam $E_{\Phi}(t)$ is shown as a solid line and the signal, $E_{\text {meas }}(t)$, with a $2 \mu \mathrm{m}$ thick Mylar film (dashed line).

For the interferometer, the measured frequency dependent signal, $E_{\text {ref }}(\omega)+E_{\text {samp }}(\omega)$ can be written as

$$
\begin{aligned}
E_{\text {meas }}(\omega)= & E_{\text {ref }}(\omega)\left\{1-\exp \left(-i k_{0}[n(\omega)-1] d\right.\right. \\
& +i \Phi(\omega))\},
\end{aligned}
$$

where we have assumed the index and absorption of the sample are small so that $t_{1 S} \cong t_{S 1} \cong 1{ }^{6}$ The phase term, $\Phi(\omega)$, accounts for both the $10.3^{\circ}$ phase difference as well as a frequency dependent term accounting for small misalignments of the sample arm. $\Phi(\omega)$ is determined by taking a scan with no sample in the interferometer, $E_{\Phi}(\omega)=E_{\text {ref }}(\omega)$ $\times\left(1-e^{i \Phi(\omega)}\right)$. Measurements of the reference arm pulse, $E_{\text {ref }}(\omega)$, and the interferometer signal with, $E_{\text {meas }}(\omega)$, and without, $E_{\Phi}(\omega)$, a sample permit determination of the complex refractive index:

$$
n_{r}(\omega)-i n_{i}(\omega)=\frac{-i}{k_{0} d} \ln \left(\frac{E_{\text {meas }}(\omega)-E_{\Phi}(\omega)}{E_{\Phi}(\omega)-E_{\text {ref }}(\omega)}+1\right)+1 .
$$

For thin samples of low index, $k_{0}[n(\omega)-1] d \ll 1$ which permits expanding the exponential in Eq. (3) as a series, ${ }^{6}$ $\exp (x) \cong 1+x$. The complex index can then be calculated by dividing the measured signal by the reference when the phase shift, $\Phi(\omega)$, is known: 


$$
\frac{E_{\text {meas }}(\omega)}{E_{\text {ref }}(\omega)} \cong k_{0} n_{i}(\omega) d+i k_{0}\left[n_{r}(\omega)-1\right] d+i \Phi(\omega) .
$$

$\mathrm{THz}$ interferometry is capable of determining small changes in the film thickness-index product directly from the time-domain data without measurement of the full $\mathrm{THz}$ pulse. The effect of a film of index, $n=n_{0}+\Delta n(\omega)$ with $\Delta n(\omega) \ll n_{0}$ and $\alpha L \ll 1$, and thickness, $d$, is to shift the THz pulse in time by an amount $\Delta t=d(n-1) / c$ for a single pass of the THz pulse through the film and $\Delta t=2 d(n-1) / c$ for a double pass. In this case, with no sample in the beam, the measured electric field in the reference arm given by $E_{\text {ref }}(t)$ and that of the sample $\operatorname{arm} E_{\text {samp }}(t)=-E_{\text {ref }}(t)+E_{\Phi}(t)$, where $E_{\Phi}(t)$ is the small residual time signal due to the phase shift, $\Phi(\omega)$. Inserting the sample shifts the pulse in the sample arm by an amount $\Delta t$. With the time shift, the measured signal is $E_{\text {meas }}(t) \cong E_{\text {ref }}(t)-E_{\text {ref }}(t-\Delta t)+E_{\Phi}(t)$. With the known $E_{\Phi}(t)$ subtracted from the data, the time shifted reference pulse can be expanded in a Taylor series with only the first order terms being retained for small $\Delta t$. In this case

$$
E_{\text {meas }}(t)=E_{\text {ref }}(t)-E_{\text {ref }}(t-\Delta t) \cong \Delta t E_{\text {ref }}^{\prime}(t),
$$

where the prime corresponds to the time derivative. The quantity $\Delta t=d(n-1) / c$ can be determined by numerically integrating $E_{\text {meas }}(t)$ and determining $\Delta t$ by a comparison to the known reference pulse. The film thickness-index product given through $\Delta t$ is directly proportional to the size of the measured signal, $E_{\text {meas }}(t)$, from the $\mathrm{THz}$ interferometer.

To demonstrate the measurement sensitivity of the interferometer we measure free standing films of nominally $2 \mu \mathrm{m}$ thick films of DuPont type C Mylar ${ }^{\circledR}$ polyester film. ${ }^{13}$ The film, stretched taut to eliminate wrinkles, was mounted on a frame placed in the sample arm of the interferometer. The film thickness, independently measured at several points on the sample. Measurements of the refractive index-thickness product of the Mylar film extracted directly from the timedomain data utilizing Eq. (6) are given in Fig. 3(a) from the data shown in Fig. 2. With both arms open and no sample in the beam, the solid line is $E_{\Phi}(t)$, due to the frequency independent phase shift and small misalignments. To find the film thickness, $E_{\Phi}(t)$ is subtracted from the measured data of Fig. 2(b), then integrated, and a value of $\Delta t$ is found which, when multiplied by the integrated data, will return the reference pulse shape. The reference pulse is shown as a solid line in Fig. 3(a) with the fit (data points) of the Mylar films using a value of $\Delta t$ of $10.5 \mathrm{fs}$, corresponding to $2 d\left(n_{r}-1\right)$ of 3.15 $\mu \mathrm{m}$. Using the published $1 \mathrm{GHz}$ dielectric constant of $\left(n_{r}\right)^{2}=\varepsilon_{r}=2.8,{ }^{13}$ this returns a value of $d=2.3 \mu \mathrm{m}$ which agrees well with the measured film thickness.

The frequency dependent index and absorption, determined from Eq. (5), are shown in Fig. 3(b). The absorption of this thin film is not measurable since the absorption length $2 \alpha L \ll 1$. The index of refraction is close to that of the published dc dielectric constant. The oscillations in the data are in part due to water vapor in the beam path. These results are in good agreement with the estimated index and film thickness obtained directly in the time domain from Fig. 3(a).
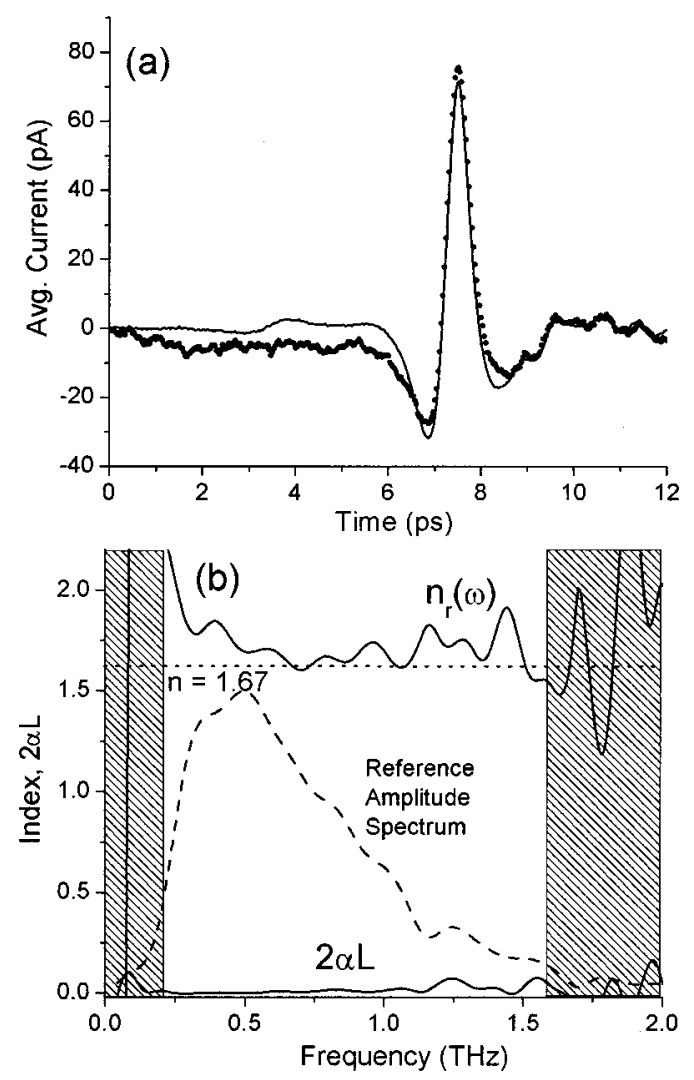

FIG. 3. (a) Measured reference pulse (solid line) $E_{\text {ref }}(t)$ and the fit of the measured pulse with background phase subtracted following Eq. (6) with $\Delta t$ of 10.5 fs. (b) Measured real refractive index (upper solid line) and absorption $\times$ length (lower solid line) determined using Eq. (5). The dotted line is the 1.67 index of Mylar at $1 \mathrm{GHz}$ while the dashed curve is the reference pulse spectral amplitude.

The authors would like to thank D. Grischkowsky for critical reading of this manuscript. This work was supported in part by the National Science Foundation and the Army Research Office.

${ }^{1}$ D. Grischkowsky, S. Keiding, M. van Exter, and C. Fattinger, J. Opt. Soc. Am. B 7, 2006 (1990).

${ }^{2}$ A. Ulman, Characterization of Organic Thin Films (ButterworthHeineman, Boston, 1995).

${ }^{3}$ D. M. Mittleman, S. Hunsche, L. Boivin, and M. C. Nuss, Opt. Lett. 22, 904 (1997).

${ }^{4}$ D. M. Mittleman, R. H. Jacobsen, and M. C. Nuss, IEEE J. Sel. Top. Quantum Electron. 2, 679 (1996)

${ }^{5}$ M. Ree, K.-J. Chen, D. P. Kirby, N. Katzenellenbogen, and D. Grischkowsky, J. Appl. Phys. 72, 2014 (1992).

${ }^{6}$ Z. P. Jiang, M. Li, and X. C. Zhang, Appl. Phys. Lett. 76, 3221 (2000).

${ }^{7}$ J. L. Johnson, T. D. Dorney, and D. M. Mittleman, Appl. Phys. Lett. 78, 835 (2001).

${ }^{8}$ N. Katzenellenbogen and D. Grischkowsky, Appl. Phys. Lett. 58, 222 (1991).

${ }^{9}$ T. I. Jeon, and D. Grischkowsky, Appl. Phys. Lett. 72, 3032 (1998).

${ }^{10}$ T. I. Jeon, D. Grischkowsky, A. K. Mukherjee, and R. Menon, Appl. Phys. Lett. 77, 2452 (2000).

${ }^{11}$ M. Born and E. Wolf, Principles of Optics, 7 th ed. (Cambridge University Press, New York, 1999).

${ }^{12}$ S. R. Keiding and D. Grischkowsky, Opt. Lett. 15, 48 (1990).

${ }^{13}$ DuPont Teijin Films, Mylar Polyester Film Electrical Properties (DuPont Teijin Films, Wilmington, DE, 1995). 